\title{
Stochastic Modelling of Insulin Sensitivity for Out of Hospital Cardiac Arrest Patients treated with Hypothermia
}

\footnotetext{
${ }^{1}$ A. Sah Pri, ${ }^{2}$ J. Geoffrey Chase, ${ }^{2}$ Christopher G. Pretty, ${ }^{3}$ Geoffrey M. Shaw, ${ }^{4}$ Jean-Charles Preiser, ${ }^{4}$ Fabio Taccone, ${ }^{5}$ Sophie Penning, ${ }^{5}$ Thomas Desaive
}

${ }^{1}$ Electrical Electronic Automation, UniKL Malaysian Spanish Institute, Kulim Kedah, Malaysia

${ }^{2}$ Center for Bio-Engineering, Mechanical Engineering, University of Canterbury, Christchurch, New Zealand.

${ }^{3}$ Christchurch School of Medical and Health Sciences, Department of Intensive Care, Christchurch, New Zealand.

${ }^{4}$ Erasme University Hospital, Department of Intensive Care, Brussels, Belgium.

${ }^{5}$ Research Unit on Bioengineering and Biophysics, GIGA-Cardiovascular Sciences, Liege, Belgium.

azurahisham@unikl.edu.my 


\section{$\underline{\text { ABSTRACT }}$}

Hypothermia is often used to treat out of hospital cardiac arrest (OHCA) patients who often simultaneously receive insulin for stress induced hyperglycaemia. Variations in response to insulin reflect dynamic changes in insulin sensitivity $\left(S_{\mathrm{I}}\right)$, defined by the overall metabolic response to stress and therapy. Thus, tracking and forecasting this parameter is important to provide safe glycaemic control in highly dynamic patients. This study examines stochastic forecasting models of model-based $S_{I}$ variability in OHCA patients to assess the resulting potential impact of this therapy on glycaemic control quality and safety. A retrospective analysis of clinically validated model-based $S_{\text {I }}$ profiles identified using data from 240 postcardiac arrest patients (9988 hours) treated with hypothermia, shortly after admission in the Intensive Care Unit (ICU). Data were divided into three periods: 1$) \operatorname{cool}\left(\mathrm{T} \geq 35^{\circ} \mathrm{C}\right)$; 2) idle period of 2 hours as hypothermia was removed; and 3) warm $\left(\mathrm{T} \geq 37^{\circ} \mathrm{C}\right)$. The stochastic model captured $60.7 \%$ and $90.2 \%$ of $S_{\text {I }}$ predictions within the (25th-75th) and (5th-95th) probability forecast intervals during cool period. Equally, it is also recorded $62.8 \%$ and $92.1 \%$ of $S_{\text {I }}$ predictions respectively during the warm period. Maintaining the kernel density variance estimator to $\mathrm{c}=1.0$ yielded $60.7 \%$ and $90.2 \%$ for the cool period. Similarly, adjusting a variance estimator of $\mathrm{c}=2.0$ yields $60.4 \%$ and $90.1 \%$ for the warm period. A cohort-specific stochastic model of $\mathrm{S}_{\text {I }}$ provided a conservative forecast for the inter-quartile range and was relatively exact for the $90 \%$ range. Adjusting the variance estimator provides a more accurate, cohort-specific stochastic model of $\mathrm{S}_{\mathrm{I}}$ dynamics for the $90 \%$ range. These latter results show clearly different levels and distribution of forecasted $S_{I}$ variability between the cold and warm periods. 


\section{INTRODUCTION}

Hyperglycaemia is prevalent in critical care (Capes et al. 2000; McCowen, Malhotra et al. 2001; Mizock 2001; van den Berghe et al. 2001) and increases the risks of further complications and mortality (Capes et al. 2000; van den Berghe et al. 2001; Krinsley 2003). Glycaemic control has shown benefits in reducing mortality (van den Berghe et al. 2001; Krinsley 2004; Chase et al. 2008). However, due in parts to excessive metabolic variability (Chase et al. 2011), other studies have found it difficult to reproduce these results (Brunkhorst et al. 2008; Finfer et al. 2009; Preiser et al. 2009) . Out-of-Hospital Cardiac Arrest (OHCA) patients have low survival rates and often experience hyperglycaemia (Taylor et al. 1994; Neumar et al. 2008). However, cardiac patients are one group who have more consistently shown benefit from glycaemic control (GC), but can be highly insulin resistant and variable, particularly on the first day of stay (Pretty et al. 2012).

Hypothermia is often used to treat OHCA patients (Neumar et al. 2008). In general, it leads to a lowering of metabolic rate that induces changes in energy metabolism. However, its impact on metabolism and insulin resistance in critical illness is unknown, although one of the adverse events associated with hypothermic therapy is a decrease in insulin sensitivity and insulin secretion (Hayashi 2009). However, this decrease may not be notable in a cohort that is already highly resistant and variable (Pretty et al. 2012). Hence, understanding metabolic evolution and variability would enable safer, more accurate GC in this cohort.

Model-based glycaemic control methods using both insulin and/or nutrition modulation have been employed successfully in the control of hyperglycaemia. These methods allow the derivation of patient metabolic state, $S_{I}$ in this case, by using serial blood glucose (BG) 
measurements, and records of nutrition and insulin administration (Chase et al. 2007) . Once the current $S_{I}$ has been identified, prediction of future $S_{I}$ would allow predictions of outcome BG concentration for an intended clinical intervention (Lin et al. 2008).

Variations in the $S_{I}$ parameter reflect the metabolic response to stress (McCowen et al. 2001) and drug therapy (Pretty et al. 2011). Thus, tracking and forecasting this parameter is important to provide safe glycaemic control in the highly dynamic Out-of-Hospital Cardiac Arrest (OHCA) patients, treated with hypothermia. Since stochastic modeling has shown its ability to quantify the probability of a future $S_{I}$ (Lin et al. 2008), the resulting distribution of BG concentrations that would result from a given intervention can be determined (Lin et al. 2008; Le Compte et al. 2010; Evans et al. 2011; Fisk et al. 2012). This information can be used to guide both insulin and/or nutrition interventions, which is the key to avoid unintended hypoglycaemia, improve overall glycaemic control, and identify periods of potential high glucose variability that may be indicative of unusual clinical events or cohorts (Thomas et al. 2014).

This paper presents the adaptation of a stochastic model for $S_{I}$ prediction from adult critical care to the unique clinical and physiological case of OHCA patients, treated with hypothermia. The stochastic model presented is constructed by the distribution of insulin sensitivity variation using a 2-D kernel density method. Clinically validated, model-based insulin sensitivity $\left(S_{I}\right)$ (Chase et al. 2010; Evans et al. 2011) is used to provide more accurate measure of patient metabolic state and its stochastic model during cool and warm periods. Modifications to the initial kernel density estimation model are made to explore and optimize the relationship between the model and the underlying dataset, particularly for the clinically 
relevant $90 \%$ range that can be used to quantify and minimize the risks of hyper- or hypoglycaemia as an integral part of a control protocol (Evans et al. 2011; Fisk et al. 2012) . 


\section{MATERIAL AND METHODS}

\section{$\underline{\text { Patients and Data }}$}

A retrospective analysis of glycaemic control data from 240 OHCA patients (9988 hours) treated with hypothermia, shortly after admission to the Intensive Care Unit (ICU) at Christchurch Hospital, New Zealand, Erasme Hospital, Belgium and Lausanne Hospital, Switzerland. Patients from Christchurch Hospital (20) were on the SPRINT glycaemic control protocol (Chase, Shaw et al. 2008), whereas the remaining 160 patients from Erasme (82) and Lausanne (78) Hospitals were on unpublished local glycaemic protocols. Blood glucose (BG) and temperature readings were taken 1-2 hourly along with all insulin and nutrition data. Data were divided into three periods: 1) cool $\left.\left(\mathrm{T} \leq 35^{\circ} \mathrm{C}\right) ; 2\right)$ transition period of 2 hours as hypothermia was removed; and 3) warm after $\left(\mathrm{T} \geq 37^{\circ} \mathrm{C}\right)$. A maximum of 24 hours and a minimum of 15 hours for each period (1 and 3) were considered, ensuring a balance of contiguous data between periods. Overall demographics are shown in Table 1.

Table 1: Demographic data for all patients.

\begin{tabular}{l|c|c}
\hline \multirow{2}{*}{ Variable } & \multicolumn{2}{c}{ Value } \\
\cline { 2 - 3 } & Cool & Warm \\
\hline Total patients, number (n) & 240 & 240 \\
\hline Total treatment, hours (h) & 4987 & 5001 \\
\hline Blood Glucose (mmol/L) [IQR] & $7.40[6.20-9.70]$ & $6.56[5.61-7.78]$ \\
\hline Insulin Rate, rate (U/hr) [IQR] & $3.37[1.33-8.00]$ & $3.51[1.60-7.00]$ \\
\hline Glucose Rate, rate (g/hr) [IQR] & $2.69[1.04-5.26]$ & $5.41[2.71-8.11]$ \\
\hline
\end{tabular}

Data are presented as median [interquartile range] where appropriate. 


\section{Therapeutic hypothermia}

Therapeutic Hypothermia (TH) was applied following a standardized written protocol. All patients were treated with mild $\mathrm{TH}$ to $33 \pm 1{ }^{\circ} \mathrm{C}$ for up to 24 hours, irrespective of age, initial arrest rhythm and other physiological conditions. TH was started immediately after admission, and was induced with ice-cold packs and/or intravenous cold fluids. Body temperature was maintained at hypothermia using a surface cooling device with a computerized adjustment of patient temperature target. During this time, some short-acting drugs, such as midazolam $(0.1 \mathrm{mg} / \mathrm{kg} . \mathrm{hr})$, fentanyl $(1.5 \mu \mathrm{g} / \mathrm{kg} / \mathrm{hr})$ and vecuronium $(0.1 \mathrm{mg} / \mathrm{kg}$ boluses), were used to administer sedation, analgesia and control shivering. Rewarming was achieved passively, and sedation-analgesia was typically stopped when patient temperature was greater than $35^{\circ} \mathrm{C}$.

\section{The Metabolic System Model}

Model-based insulin sensitivity $\left(S_{I}\right)$ in this study is a patient-specific parameter describing the whole body effect of insulin. The analysis of patient-specific insulin sensitivity employs the ICING model (Lin et al. 2011) as a glucose-insulin system model developed and clinically validated in critical care glycaemic control and insulin sensitivity studies (Chase et al. 2010; Evans et al. 2011; Lin et al. 2011; Fisk et al. 2012). It is defined:

$$
\begin{aligned}
& \dot{G}=-p_{G} \cdot G(t)-S_{I} \cdot G(t) \cdot \frac{Q(t)}{1+\alpha_{G} Q(t)}+\frac{P(t)+E G P_{b}-C N S}{V_{G}} \\
& \dot{I}=-n_{K} I(t)-\frac{n_{L} I(t)}{1+\alpha_{I} I(t)}-n_{I}(I(t)-Q(t))+\frac{u_{e x}(t)}{V_{I}}+\left(1-X_{L}\right) \frac{u_{e n}(G)}{V_{I}} \\
& \dot{Q}=n_{I}(I(t)-Q(t))-n_{C} \frac{Q(t)}{1+\alpha{ }_{G} Q(t)}
\end{aligned}
$$




$$
\begin{aligned}
& \dot{P}_{1}=-d_{1} P_{1}+D(t) \\
& \dot{P}_{2}=-\min \mathbb{X}_{2} P_{2}, P_{\max } \exists d_{1} P_{1} \\
& P(t)=\min \mathbb{E}_{2} P_{2}, P_{\max } \exists P(t) \\
& u_{e n}(G)=\min \operatorname{Aax}\left(u_{\min }, k_{1} G(t)+k_{2}\right), u_{\max }-
\end{aligned}
$$

\begin{tabular}{|c|c|c|c|c|}
\hline Parameter & \multicolumn{2}{|c|}{ Value } & Unit & Description \\
\hline $\mathrm{p}_{\mathrm{G}}$ & \multicolumn{2}{|c|}{0.006} & $\min ^{-1}$ & Non-insulin mediated glucose removal \\
\hline EGP & \multicolumn{2}{|c|}{1.16} & $\mathrm{mmol} / \mathrm{min}$ & Endogenous glucose production rate \\
\hline CNS & \multicolumn{2}{|c|}{0.3} & $\mathrm{mmol} / \mathrm{min}$ & Central nervous system glucose uptake \\
\hline $\mathrm{V}_{\mathrm{G}}$ & \multicolumn{2}{|c|}{13.3} & $\mathrm{~L}$ & Plasma glucose distribution volume \\
\hline $\mathrm{V}_{\mathrm{I}}$ & \multicolumn{2}{|c|}{4.0} & $\mathrm{~L}$ & $\begin{array}{l}\text { Plasma and interstitial insulin distribution } \\
\text { volume }\end{array}$ \\
\hline$\alpha_{\mathrm{G}}$ & \multicolumn{2}{|c|}{0.0154} & $\mathrm{~L} / \mathrm{mU}$ & Insulin binding saturation parameter \\
\hline$\alpha_{\mathrm{I}}$ & \multicolumn{2}{|c|}{0.0017} & $\mathrm{~L} / \mathrm{mU}$ & $\begin{array}{l}\text { Hepatic insulin clearance saturation } \\
\text { parameter }\end{array}$ \\
\hline $\mathrm{n}_{\mathrm{I}}$ & \multicolumn{2}{|c|}{0.006} & $\min ^{-1}$ & Trans-endothelial diffusion rate \\
\hline $\mathrm{n}_{\mathrm{C}}$ & \multicolumn{2}{|c|}{0.006} & $\min ^{-1}$ & Interstitial insulin degradation rate \\
\hline $\mathrm{n}_{\mathrm{K}}$ & \multicolumn{2}{|c|}{0.0542} & $\min ^{-1}$ & Renal insulin clearance rate \\
\hline $\mathrm{n}_{\mathrm{L}}$ & \multicolumn{2}{|c|}{0.1578} & $\min ^{-1}$ & Hepatic insulin clearance rate \\
\hline $\mathrm{X}_{\mathrm{L}}$ & \multicolumn{2}{|c|}{0.67} & & $\begin{array}{l}\text { Fractional first-pass } \\
\text { extraction }\end{array}$ hepatic insulin \\
\hline $\mathrm{d}_{1}$ & \multicolumn{2}{|c|}{0.0347} & $\min ^{-1}$ & Glucose transport rate from stomach to gut \\
\hline $\mathrm{d}_{2}$ & \multicolumn{2}{|c|}{0.0069} & $\min ^{-1}$ & Glucose transport rate from gut to plasma \\
\hline $\mathrm{P}_{\max }$ & \multicolumn{2}{|c|}{6.11} & $\mathrm{mmol} / \mathrm{min}$ & Maximum glucose flux from gut to plasma \\
\hline $\mathrm{u}_{\min }$ & \multicolumn{2}{|c|}{16.7} & $\mathrm{mU} / \mathrm{min}$ & Minimum pancreatic secretion rate \\
\hline $\mathrm{u}_{\max }$ & \multicolumn{2}{|c|}{266.7} & $\mathrm{mU} / \mathrm{min}$ & Maximum pancreatic secretion rate \\
\hline \multirow[t]{3}{*}{$\mathrm{k}_{1}$} & ND & 14.9 & \multirow[t]{3}{*}{ mU.L/mmol.min } & \multirow{3}{*}{$\begin{array}{l}\text { Pancreatic insulin secretion glucose- } \\
\text { sensitivity }\end{array}$} \\
\hline & T2DM & 4.9 & & \\
\hline & T1DM & 0.0 & & \\
\hline \multirow[t]{3}{*}{$\mathrm{k}_{2}$} & ND & -49.9 & \multirow[t]{3}{*}{$\mathrm{mU} / \mathrm{min}$} & \multirow[t]{3}{*}{ Pancreatic insulin secretion offset } \\
\hline & T2DM & -27.4 & & \\
\hline & T1DM & 16.7 & & \\
\hline
\end{tabular}

A summary of parameter values and descriptions, and exogenous input variables for the model are listed in Tables 2 and 3 respectively.

Table 2: Parameter values and descriptions for the ICING model 
Table 3: Exogenous model input variables

\begin{tabular}{|c|c|l|}
\hline Variable & Unit & \multicolumn{1}{c|}{ Description } \\
\hline $\mathrm{PN}(\mathrm{t})$ & $\mathrm{mmol} / \mathrm{min}$ & Intravenous glucose input rate (parenteral nutrition) \\
\hline $\mathrm{D}(\mathrm{t})$ & $\mathrm{mmol} / \mathrm{min}$ & Oral glucose input rate (enteral nutrition) \\
\hline $\mathrm{u}_{\mathrm{ex}}(\mathrm{t})$ & $\mathrm{mU} / \mathrm{min}$ & Intravenous insulin input rate \\
\hline
\end{tabular}

Insulin sensitivity $S_{I}$ is identified hourly from patient data, producing a step-wise hourly varying profile (Hann et al. 2005). This profile effectively describes patient-specific metabolic behavior under time-varying physiologic conditions. The validity and independence of this patient-specific parameter have been validated using data from independent, clinically matched cohorts (Chase et al. 2010) and in gold-standard insulin sensitivity tests (McAuley et al. 2011).

\section{The Stochastic Model}

A 2-D kernel density estimation method is used to construct the stochastic model that describes the hourly transition of $S_{I}$. The kernel density method combines probability distribution functions for each point of data to generate an overall density function for the dataset. This method has the advantage of producing a smooth, physiologically likely, continuous function across the parameter range to provide continuity when interpolating $S_{I}$ forecasts to account for each particular patient state. It also automatically accounts for any possible multimodality where the density of data may show several distinct peaks corresponding to patterns of changes in $S_{I}$. The overall result is a bivariate probability density function for the potential parameter values. The goal of this statistical model is to quantify the range of $S_{I}$ one hour ahead in time $\left(S_{I, n+1}\right)$ based on available data $\left(S_{I, n}, S_{I, n-1}, S_{I, n-2}, \ldots\right.$, $S_{I, 0}$ ) to guide real-time clinical control (Evans, Shaw et al. 2011; Fisk, Le Compte et al. 2012). Thus, it is potentially important that the model is also as cohort-specific as possible for greatest accuracy and to minimize over-conservative forecasts (Thomas et al. 2014). 
A 2-D kernel density method is chosen because the distribution of $S_{I, n+l}$ varies with $S_{I, n}$, and cannot be simply described with a single standard statistical distribution. Thus, the variations in $S_{I}$ can be treated as a Markov process. A Markov process has the property that the conditional probability density function of future states of the process, given the current state, depends only upon the current state. Therefore, using the Markov property of the stochastic behaviour of $S_{I}$, the conditional probability density of $S_{I, n+1}$ taking on a value y can be calculated by knowing $S_{I, n}=x$. Model equations and derivation were defined in (Lin et al. 2008) .

In this study, the model was cross validated by splitting the 240 of cool and warm patient cohorts into five groups, each containing 48 patients per period following the method of (Lin et al. 2008). For each group, the model created using the remaining 192 patients of the cohort representing approximately 3990 hours of data per period. Out-of-sample $S_{\text {I }}$ predictions were generated for the 48 patients of unused group and compared to the actual fitted $S_{I}$ from these 48 patients to assess model accuracy and cohort generality. Based on results from in-sample tests, where the stochastic model is generated from the entire retrospective dataset and tested on the same data, and out-of-sample tests, where different subsets of data are used for model generation and testing, the kernel density estimator was modified by multiplying the variance estimators by a constant $\mathrm{c}$ (i.e., $c \sigma_{\mathrm{x}}$ and $c \sigma_{\mathrm{y}}$ ) to explore the model bias-variance trade-off for this data between cool and warm periods as well as 6 and 12 hour blocks. This adjustment to the variance estimator effectively adjusts the kernel bandwidth and the degree of smoothing over the data, which is used here to assess variability differences between periods or cohorts. 


\section{Analyses and Metrics}

Current $\mathrm{S}_{\mathrm{I}}(\mathrm{n})$ during the cool $\left(\mathrm{T} \leq 35^{\circ} \mathrm{C}\right)$ and warm $\left(\mathrm{T} \geq 37^{\circ} \mathrm{C}\right)$ periods were identified hourly using the ICING model (Lin et al. 2011) for each patient. Using current $S_{I}$ (n) data, the predicted $S_{I}(n+1)$ data can be generalized by shifting 1-hour forward from the current $S_{I}(n)$ data. These $S_{I}$ vectors will be paired and used to create the stochastic $S_{I}$ model of OHCA cohort. Stochastic $S_{I}$ models were analyzed during both cool and warm periods as follows:

i) Overall cohort patient.

ii) Analysis of patients in 6-hour block.

Overall cohort analysis assessed the stochastic model behavior of insulin sensitivity during both cool and warm periods, which includes percentage of $S_{I}$ within prediction interval and analysis of modifying kernel density estimation. $\mathrm{S}_{\mathrm{I}}$ is also analyzed using 6-hour blocks, as described in Table 4, to capture $S_{I}$ variability and forecasting over time with different resolution. The analysis includes percentage of $S_{I}$ within predicted interval and analysis of modifying the kernel density estimation.

Table 4: Descriptions of 6-hour blocks for data analysis

\begin{tabular}{|c|c|c|c|}
\hline \multirow{2}{*}{ Day } & \multicolumn{3}{|c|}{ 6-hour blocks } \\
\cline { 2 - 4 } & Block & Hours Range & Period \\
\hline \multirow{4}{*}{1} & 1 & $0-6$ hours & Cool \\
\cline { 2 - 4 } & 2 & $6-12$ hours & Cool \\
\cline { 2 - 4 } & 3 & $12-18$ hours & Cool \\
\cline { 2 - 4 } & 4 & $18-24$ hours & Cool \\
\hline \multirow{4}{*}{2} & 5 & $24-30$ hours & Warm \\
\cline { 2 - 4 } & 6 & $30-36$ hours & Warm \\
\cline { 2 - 4 } & 7 & $36-42$ hours & Warm \\
\cline { 2 - 4 } & 8 & $42-48$ hours & Warm \\
\hline
\end{tabular}




\section{RESULTS AND DISCUSSIONS}

\section{Distribution of hourly variation in $\mathrm{S}_{\mathrm{I}}$}

Figure 1 presents the distribution of hourly variation in $S_{I}$ and the stochastic model percentile probability bands at $\mathrm{c}=1.0$, for the 240 OHCA patients during cool (4987 hours) and warm (5001 hours) periods. Approximately $85 \%$ of the values during cool period, and $70 \%$ during warm period are below $1.0 \times 10^{-3} \mathrm{~L} /(\mathrm{mU} . \mathrm{min})$. The results show that the hourly variation of $S_{I}$ is wider during the cool period, and $S_{I}$ rises from cool to warm.

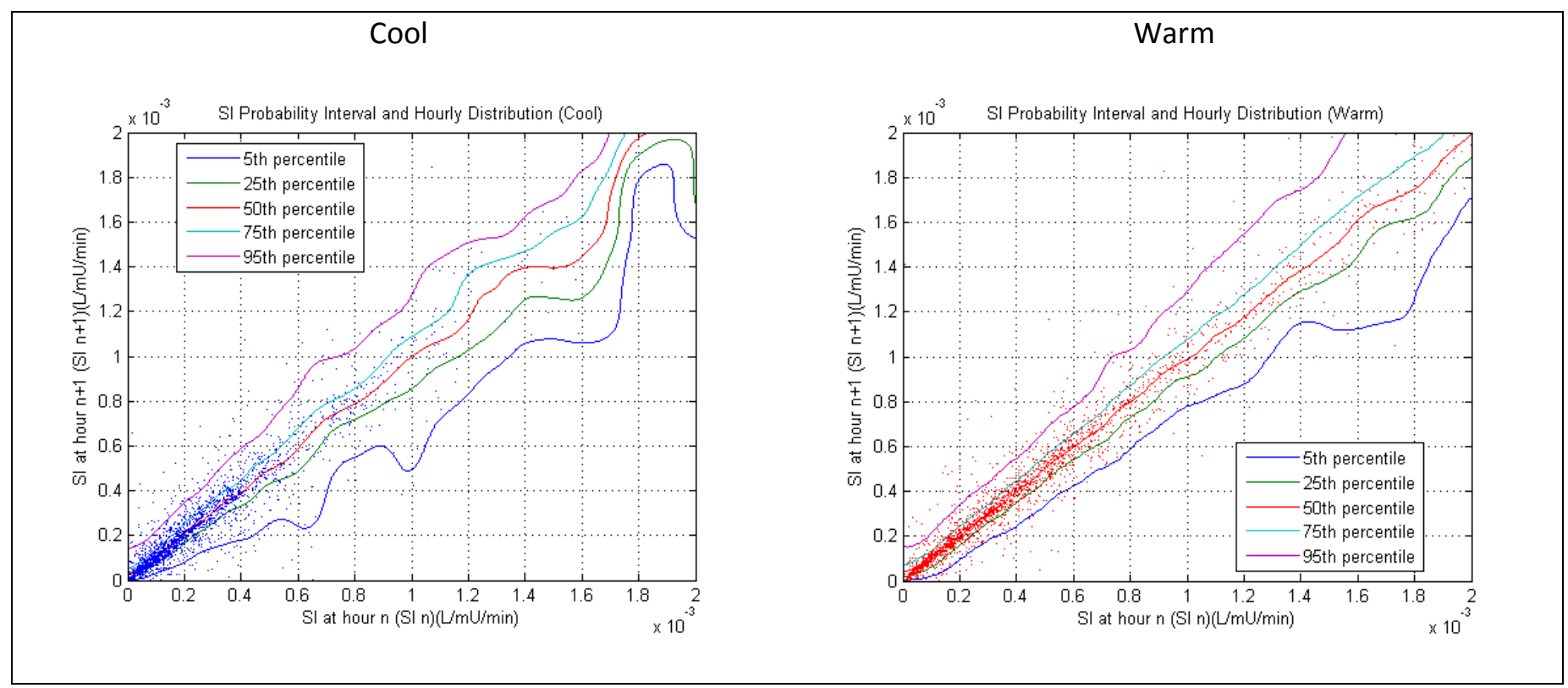

Fig. 1: Probability interval and distribution of hourly variation in $S_{I}$ for $O H C A$ patients, treated with hypothermia during cool (left) and warm (right) periods.

Table 5 shows the in-sample results of stochastic model prediction widths for both cool $\left(\mathrm{n}_{\text {cool }}\right.$ $=4622$ predictions $)$ and warm $\left(\mathrm{n}_{\mathrm{warm}}=4832\right.$ predictions $)$ periods. The number of predictions is less than the total hours of $S_{I}$ as the patient data records are not always perfectly divisible by $1 \mathrm{~h}$, and because predictions can only be computed after the second hour of patient data. 
For the cool period, $S_{\text {I }}$ predictions $(60.7 \%)$ were within the (25th-75th) probability intervals, and within the (5th-95th) probability interval. Thus, the proportion of $S_{\text {I }}$ values that fell within the forecast (25th-75th) probability intervals were measurably higher than the expected $50 \%$, but very much closer for the $90 \%$ intervals. Similar results could be observed for the warm period, but were measured higher than cool period for both $50 \%$ and $90 \%$ intervals.

Table 5: Sample results for in-sample stochastic model prediction widths at $c=1$

\begin{tabular}{|l|l|l|l|}
\hline \multirow{2}{*}{ Variable } & \multirow{2}{*}{$\begin{array}{l}\text { Prediction width } \\
\text { / Range }\end{array}$} & Value \\
\cline { 3 - 4 } & {$\left[25^{\text {th }}-75^{\text {th }}\right]$} & $60.7 \%$ & $62.8 \%$ \\
\hline $\begin{array}{l}\text { \% SI within } \\
\text { prediction interval }\end{array}$ & {$\left[5^{\text {th }}-95^{\text {th }}\right]$} & $90.2 \%$ & $92.1 \%$ \\
\hline
\end{tabular}

The percentage SI within prediction interval results of Lin et al. of $54.0 \%$ is within the $(25 \mathrm{th}-$ 75th) probability bound shows that normal adult ICU patients with normal body temperature produce far closer to the ideal $50 \%$. However, the prediction interval percentage results of $60.7 \%$ and $62.8 \%$ at cool and warm respectively on the same probability bound for the OHCA patients, treated with hypothermia as shown in Table 5 appear to be unique and significant. These results are similar to neonates' results of (Le Compte et al. 2010), which record $62.6 \%$ at the same interval. 


\section{Cross-Validation Comparison Studies}

Table 6 shows the results of the cross validation comparison study for 240 patients' cohort during the cool and warm periods, respectively. Generally, these results are consistent between groups, suggesting that the overall model contains sufficient data to account for the range of dynamics observed in this cohort.

Table 6: Cross-validation comparison study for 240 patient cohort

\begin{tabular}{|c|c|c|c|c|c|}
\hline Group & \multirow{2}{*}{$\begin{array}{c}\text { Groups used } \\
\text { to create the } \\
\end{array}$} & \multicolumn{2}{|c|}{$\begin{array}{c}\text { \% SI within interval during } \\
\text { model }\end{array}$} & $\begin{array}{c}\text { \% SI within interval during } \\
\text { warm period }\end{array}$ & \multicolumn{2}{|c|}{\begin{tabular}{c} 
cool ped \\
\cline { 3 - 6 }
\end{tabular}} & {$\left[25^{\text {th }}-75^{\text {th }}\right]$} & {$\left[5^{\text {th }}-95^{\text {th }}\right]$} & {$\left[25^{\text {th }}-75^{\text {th }}\right]$} & {$\left[5^{\text {th }}-95^{\text {th }}\right]$} \\
\hline 1 & {$[-, 2,3,4,5]$} & 60.1 & 90.5 & 64.1 & 92.3 \\
\hline 2 & {$[1,-, 3,4,5]$} & 61.2 & 90.0 & 64.0 & 92.6 \\
\hline 3 & {$[1,2,-, 4,5]$} & 61.9 & 90.8 & 61.7 & 91.8 \\
\hline 4 & {$[1,2,3,-, 5]$} & 61.9 & 90.8 & 63.1 & 92.7 \\
\hline 5 & {$[1,2,3,4,-]$} & 62.6 & 90.7 & 63.8 & 92.8 \\
\hline Overall & {$[\mathbf{1 , 2 , 3 , 4 , 5}]$} & $\mathbf{6 0 . 7}$ & $\mathbf{9 0 . 2}$ & $\mathbf{6 2 . 8}$ & $\mathbf{9 2 . 1}$ \\
\hline
\end{tabular}

\section{Probability-bound determination using local variance estimator}

Table 7 shows the effect of modifying the kernel density estimation for several values of $\mathrm{c}$, ranging from 0.1 to 3.0. For this cohort, the increase of $\mathrm{c}>1.0$, yield better coverage widths for the expected proportions. Thus, for cool period, the value $\mathrm{c}=1.0$ enables the best $90 \%$ interval coverage, but is conservative for the inter-quartile range. Similarly, $c=2.0$ is best for the warm period. 
Table 7: Comparison of probability bounds for modifications of kernel density estimator $\left(\sigma^{\prime}{ }_{\mathrm{x}}\right.$ $=c \sigma_{\mathrm{x}} \mathrm{AND}^{\prime}{ }_{\mathrm{y}}=\mathrm{c} \sigma_{\mathrm{y}}$ ) during both cool and warm periods.

\begin{tabular}{|c|c|c|c|c|}
\hline \multirow{2}{*}{ C } & \multicolumn{2}{|c|}{$\begin{array}{c}\text { \% of SI within probability bound } \\
\text { during Cool period }\end{array}$} & \multicolumn{2}{c|}{$\begin{array}{c}\text { of SI within probability bound } \\
\text { during Warm period }\end{array}$} \\
\cline { 2 - 5 } & {$\left[25^{\text {th }}-75^{\text {th }}\right]$} & {$\left[5^{\text {th }}-95^{\text {th }}\right]$} & {$\left[25^{\text {th }}-75^{\text {th }}\right]$} & {$\left[5^{\text {th }}-95^{\text {th }}\right]$} \\
\hline 0.1 & 49.3 & 87.8 & 45.5 & 83.7 \\
\hline 0.2 & 50.2 & 88.4 & 48.2 & 84.4 \\
\hline 0.3 & 51.1 & 88.5 & 50.4 & 85.2 \\
\hline 0.5 & 53.5 & 88.6 & 52.2 & 85.9 \\
\hline 1.0 & $\mathbf{6 0 . 7}$ & $\mathbf{9 0 . 2}$ & 55.8 & 88.9 \\
\hline 1.5 & 66.2 & 91.3 & 58.0 & $\mathbf{9 0 . 1}$ \\
\hline 2.0 & 69.6 & 92.1 & $\mathbf{6 0 . 4}$ & 91.2 \\
\hline 2.5 & 72.2 & 92.7 & 62.8 & 91.5 \\
\hline 3.0 & 74.0 & 93.4 & 64.9 & $\mathbf{9 0 \%}$ \\
\hline Ideal & $\mathbf{5 0 \%}$ & $\mathbf{9 0 \%}$ & $\mathbf{5 0 \%}$ & \\
\hline
\end{tabular}

The difference of optimal c values between cool and warm suggests that the variation and stochastic modeling for both periods are different, leading to potentially different control requirements to ensure safe glycaemic control in these highly dynamic patients and states. In particular, c=1.0 is currently used in the STAR protocol (Evans et al. 2012; Penning et al. 2012) in Medical ICU patients. Hence, no change is required for the cool period. However, during the warm period, the stochastic model requires a wider and smoother probability distribution coverage at $c=2.0$, which would necessitate a control change of this known change of state. These results are further reflected in Figure 2. 


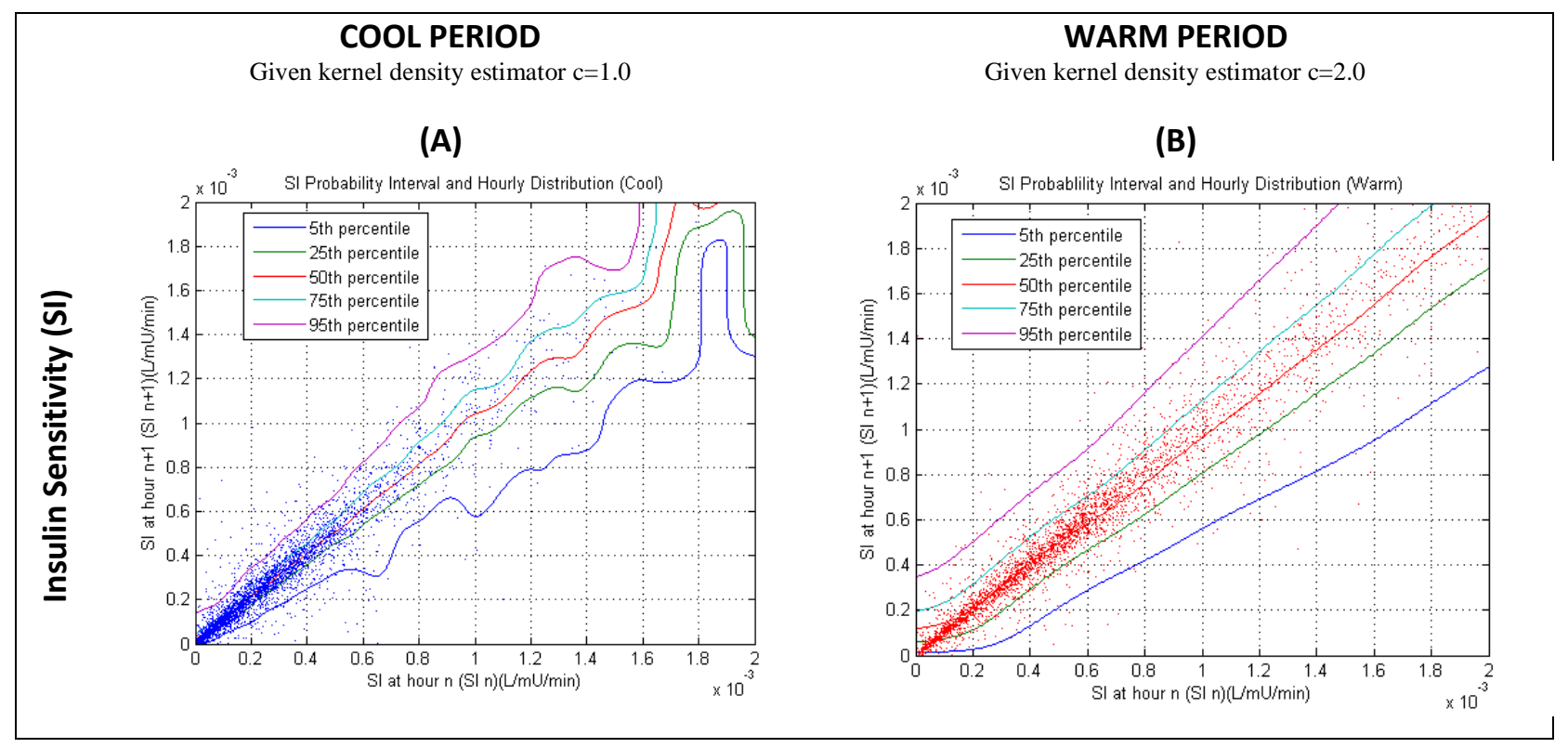

Fig. 2: Probability-bound determination for raw $S_{I}$ data and corresponding $B G$ forecasted values based on an equal-tailed 0.90 probability interval of $S_{I}$ between $5^{\text {th }}-95^{\text {th }}$ at local variance estimator, both cool ( $\mathrm{c}=1.0)$ and warm $(\mathrm{c}=2.0)$ period. The solid lines represent the $5 \%, 25 \%, 50 \%, 75 \%$ and $95 \%$ probability bounds.

The kernel density estimator method employed in this stochastic model provides a layer of safety as wider probability bounds would be more likely to capture dynamics and any changes not observed in the cohort. As the (5th - 95th) band is what has been used for control previously, these cohorts show that they are closer to ideal $90 \%$. However, wider coverage bands may also have impact on glycaemic control performance. As the wider probability band might be useful to avoid potential hypoglycaemia, it may also force a controller to maintain a mildly hyperglycaemic state. Importantly, different level of smoothing are required for the cool and warm periods, indicating that cool patients are less multi-modal (more smoothed) and warm OHCA patients are more multi-modal (less smoothed), in comparison to broad ICU cohorts. Perhaps this scenario is caused by the suppression of metabolic activities during cool period. 


\section{Stochastic Analysis by 6-hour block}

The analysis of stochastic model based on 6-hour time block have used the same methodology as the overall cohort patient, except that the patient cohort data are separated in block hours. Table 8 presents the in-sample results of stochastic model prediction widths based on 6 hour block analysis at $\mathrm{c}=1.0$.

Table 8: Stochastic model prediction widths based on 6-hour block analysis

\begin{tabular}{|c|c|c|c|c|c|c|c|c|c|}
\hline \multirow{2}{*}{ Variable } & \multirow{2}{*}{$\begin{array}{c}\text { Prediction } \\
\text { width / }\end{array}$} & \multicolumn{4}{|c|}{ Cool } & \multicolumn{4}{c|}{ Warm } \\
\cline { 3 - 10 } & Range & $\begin{array}{c}\text { Block 1 } \\
{[0-6 \mathrm{hrs}]}\end{array}$ & $\begin{array}{c}\text { Block 2 } \\
{[6-12} \\
\mathrm{hrs}]\end{array}$ & $\begin{array}{c}\text { Block 3 } \\
{[12-18} \\
\mathrm{hrs}]\end{array}$ & $\begin{array}{c}\text { Block 4 } \\
{[18-24} \\
\mathrm{hrs}]\end{array}$ & $\begin{array}{c}\text { Block 5 } \\
{[24-30} \\
\mathrm{hrs}]\end{array}$ & $\begin{array}{c}\text { Block 6 } \\
{[30-36} \\
\mathrm{hrs}]\end{array}$ & $\begin{array}{c}\text { Block 7 } \\
{[36-42} \\
\mathrm{hrs}]\end{array}$ & $\begin{array}{c}\text { Block 8 } \\
{[42-48} \\
\mathrm{hrs}]\end{array}$ \\
\hline $\begin{array}{c}\text { \% SI within } \\
\text { prediction } \\
\text { interval }\end{array}$ & {$\left[25^{\text {th }}-75^{\text {th }}\right]$} & 56.1 & 60.2 & 58.9 & 61.5 & 61.9 & 62.1 & 62.6 & 62.6 \\
\cline { 2 - 11 } & {$\left[5^{\text {th }}-95^{\text {th }}\right]$} & 90.1 & 91.9 & 91.0 & 91.3 & 91.2 & 92.2 & 92.3 & 92.7 \\
\hline
\end{tabular}

The results show that $S_{\text {I }}$ prediction interval coverage meets the prediction expectation despite exceeding its ideal values of $50 \%$ and $90 \%$ for both $\left(25^{\text {th }}-75^{\text {th }}\right)$ and $\left(5^{\text {th }}-95^{\text {th }}\right)$ interval width respectively. It is also observed that the proportion of fitted $S_{I}$ values that fell within the $\left(25^{\text {th }}\right.$ $\left.-75^{\text {th }}\right)$ and $\left(5^{\text {th }}-95^{\text {th }}\right)$ probability intervals for 6-hour block analysis were measured higher than the expected $50 \%$ and $90 \%$.

Table 9 shows the effect of modifying the kernel density estimation for several values of $\mathrm{c}$, ranging from 0.1 to 3.0 based on 6 hour block analysis. For this cohort, the results has shown that for block 1, $\mathrm{c}=1.0$ and followed by block 2 to block $3(\mathrm{c}=1.5)$, block 4 to block $5(\mathrm{c}=2.0)$, block 6 to block $7(\mathrm{c}=2.5)$, and block $8(\mathrm{c}=3.0)$. The trend shows that the value of estimator, $\mathrm{c}$ is increased as $S_{I}$ increases from cool to warm, and match with overall cohort stochastic 
model analysis as shown in the Table 7 . This will lead to another idea of separating stochastic model for each time block to implement stochastic control. The difference of optimal c values between 6-hour blocks suggests that the variation and stochastic modeling for each time block is different, leading to different control requirements to ensure safe glycaemic control in the highly dynamic conditions. 
Table 9: Comparison of probability bounds for modifications of kernel density estimator $\left(\sigma^{\prime}{ }_{x}=c \sigma_{x}\right.$ AND $\left.\sigma_{y}^{\prime}=\sigma_{y}\right)$ based on 6-hour block analysis

\begin{tabular}{|c|c|c|c|c|c|c|c|c|c|c|c|c|c|c|c|c|}
\hline \multirow{3}{*}{$\mathrm{C}$} & \multicolumn{8}{|c|}{ Cool Period } & \multicolumn{8}{|c|}{ Warm Period } \\
\hline & \multicolumn{2}{|c|}{$\begin{array}{c}\% \text { of } \mathrm{S}_{\mathrm{I}} \text { within } \\
\text { probability bounds } \\
\text { at Block } 1 \\
0-6] \text { hours }\end{array}$} & \multicolumn{2}{|c|}{$\begin{array}{c}\% \text { of } \mathrm{S}_{\mathrm{I}} \text { within } \\
\text { probability bounds } \\
\text { at Block } 2\end{array}$} & \multicolumn{2}{|c|}{$\begin{array}{c}\% \text { of } S_{I} \text { within } \\
\text { probability bounds } \\
\text { at Block } 3 \\
{[12-18] \text { hours }}\end{array}$} & \multicolumn{2}{|c|}{$\begin{array}{c}\% \text { of } \mathrm{S}_{\mathrm{I}} \text { within } \\
\text { probability bounds } \\
\text { at Block } 4 \\
{[18-24] \text { hours }}\end{array}$} & \multicolumn{2}{|c|}{$\begin{array}{c}\% \text { of } S_{I} \text { within } \\
\text { probability bounds } \\
\text { at Block } 5 \\
{[24-] \text { hours }}\end{array}$} & \multicolumn{2}{|c|}{$\begin{array}{c}\% \text { of } S_{I} \text { within } \\
\text { probability bounds } \\
\text { at Block } 6 \\
{[36-48] \text { hours }}\end{array}$} & \multicolumn{2}{|c|}{$\begin{array}{c}\% \text { of } \mathrm{S}_{\mathrm{I}} \text { within } \\
\text { probability bounds } \\
\text { at Block } 7 \\
{[36-48] \text { hours }}\end{array}$} & \multicolumn{2}{|c|}{$\begin{array}{c}\% \text { of } \mathrm{S}_{\mathrm{I}} \text { within } \\
\text { probability bounds } \\
\text { at Block } 8 \\
36-48] \text { hours }\end{array}$} \\
\hline & $\begin{array}{c}{\left[25^{\text {th }}-\right.} \\
\left.75^{\text {th }}\right]\end{array}$ & $\begin{array}{l}{\left[5^{\text {th }}-\right.} \\
\left.95^{\text {th }}\right]\end{array}$ & {$\left[25^{\text {th }}-\right.$} & $\begin{array}{l}{\left[5^{\text {th }}-\right.} \\
\left.95^{\text {th }}\right]\end{array}$ & {$\left[25^{\text {th }}-\right.$} & $\begin{array}{l}{\left[5^{\text {th }}-\right.} \\
\left.95^{\text {th }}\right]\end{array}$ & {$\left[25^{\text {th }}-\right.$} & $\begin{array}{l}{\left[5^{\text {th }}-\right.} \\
\left.95^{\text {th }}\right]\end{array}$ & {$\left[25^{\text {th }}-\right.$} & $\begin{array}{l}{\left[5^{\text {th }}-\right.} \\
\left.95^{\text {th }}\right]\end{array}$ & $\begin{array}{c}{\left[25^{\text {th }}-\right.} \\
\left.75^{\text {th }}\right]\end{array}$ & $\begin{array}{l}{\left[5^{\text {th }}-\right.} \\
\left.95^{\text {th }}\right]\end{array}$ & {$\left[25^{\text {th }}-\right.$} & $\begin{array}{l}{\left[5^{5^{\mathrm{th}}}-\right.} \\
\left.95^{\mathrm{th}}\right]\end{array}$ & $\begin{array}{l}25^{\text {th }}- \\
\left.75^{\text {th }}\right]\end{array}$ & {$\left[5^{\text {th }}-\right.$} \\
\hline 0.1 & 51.6 & 88.9 & 52.7 & 85.3 & 53.5 & 85.8 & 51.7 & 86.0 & 44.9 & 84.2 & 45.0 & 85.3 & 45.5 & 85.5 & 46.2 & 85.6 \\
\hline 0.2 & 51.4 & 89.3 & 53.5 & 86.2 & 54.3 & 86.6 & 52.1 & 86.2 & 45.3 & 84.5 & 45.4 & 85.8 & 46.2 & 85.8 & 46.7 & 85.9 \\
\hline 0.3 & 51.7 & 89.5 & 54.0 & 86.9 & 54.7 & 86.9 & 53.5 & 86.6 & 46.3 & 85.8 & 46.7 & 86.6 & 47.4 & 86.6 & 48.0 & 86.7 \\
\hline 0.5 & 53.1 & 89.6 & 55.6 & 88.8 & 55.1 & 87.8 & 54.3 & 87.4 & 48.4 & 86.5 & 48.6 & 87.0 & 49.2 & 87.1 & 49.3 & 87.3 \\
\hline 1.0 & 58.7 & 90.4 & 57.2 & 89.3 & 56.9 & 89.0 & 55.5 & 87.3 & 51.9 & 87.2 & 52.1 & 88.2 & 52.6 & 88.3 & 52.6 & 87.7 \\
\hline 1.5 & 62.7 & 91.0 & 58.9 & 90.7 & 58.0 & 90.6 & 57.2 & 88.2 & 54.9 & 87.8 & 55.2 & 88.6 & 55.5 & 88.8 & 55.7 & 88.3 \\
\hline 2.0 & 65.6 & 91.5 & 61.0 & 93.5 & 60.2 & 92.1 & 59.2 & 90.7 & 55.6 & 90.3 & 56.1 & 89.3 & 56.2 & 89.4 & 57.2 & 88.9 \\
\hline 2.5 & 67.3 & 92.3 & 63.4 & 94.2 & 62.7 & 92.5 & 60.8 & 92.4 & 56.2 & 91.9 & 55.8 & 90.9 & 57.3 & 90.1 & 58.5 & 89.5 \\
\hline 3.0 & 68.0 & 92.8 & 65.7 & 94.9 & 63.9 & 92.6 & 62.1 & 92.9 & 56.7 & 92.8 & 57.5 & 91.2 & 57.9 & 91.3 & 59.0 & 90.7 \\
\hline Ideal & $50 \%$ & $90 \%$ & $50 \%$ & $90 \%$ & $50 \%$ & $90 \%$ & $50 \%$ & $90 \%$ & $\mathbf{5 0 \%}$ & $90 \%$ & $50 \%$ & $90 \%$ & $\mathbf{5 0 \%}$ & $90 \%$ & $\mathbf{5 0 \%}$ & $90 \%$ \\
\hline
\end{tabular}




\section{CONCLUSION}

Overall, this stochastic method and analysis in this study provides predictions based on a cohort dataset. The prediction bounds for more dynamic patients are difficult to decide since the $S_{I}$ level and variability distribution for this cohort is unique (Sah Pri et al. 2014), and tracked its evolution over time, particularly during cool period. This observation is far differing than for the less dynamic patients who are typically more conservative. Thus, the probability bounds are optimized in a cohort sense, but not necessarily applicable on a perpatient basis.

\section{ACKNOWLEDGEMENTS}

I would like to thank my supervisory team, headed by Prof. Dr Geoff Chase, Dr Thomas Desaive, Dr. Chris Pretty, and my colleagues at the Center for Bio-Engineering, Mechanical Engineering, University of Canterbury, Christchurch, New Zealand who provided insight and expertise that greatly assisted the research. 


\section{REFERENCE}

Brunkhorst, F. M., C. Engel, et al. (2008). "Intensive insulin therapy and pentastarch resuscitation in severe sepsis." N Engl J Med 358(2): 125-139.

Capes, S. E., D. Hunt, et al. (2000). "Stress hyperglycaemia and increased risk of death after myocardial infarction in patients with and without diabetes: a systematic overview." Lancet 355(9206): 773-778.

Chase, J. G., A. J. Le Compte, et al. (2011). "Tight glycemic control in critical care--the leading role of insulin sensitivity and patient variability: a review and model-based analysis." Comput Methods Programs Biomed 102(2): 156-171.

Chase, J. G., G. Shaw, et al. (2008). "Implementation and evaluation of the SPRINT protocol for tight glycaemic control in critically ill patients: a clinical practice change." $\underline{\text { Crit }}$ Care 12(2): R49.

Chase, J. G., G. M. Shaw, et al. (2007). "Model-based insulin and nutrition administration for tight glycaemic control in critical care." Curr Drug Deliv 4(4): 283-296.

Chase, J. G., F. Suhaimi, et al. (2010). "Validation of a model-based virtual trials method for tight glycemic control in intensive care." Biomed Eng Online 9: 84.

Evans, A., A. Le Compte, et al. (2012). "Stochastic targeted (STAR) glycemic control: design, safety, and performance." J Diabetes Sci Technol 6(1): 102-115.

Evans, A., G. M. Shaw, et al. (2011). "Pilot proof of concept clinical trials of Stochastic Targeted (STAR) glycemic control." Ann Intensive Care 1: 38.

Finfer, S., D. R. Chittock, et al. (2009). "Intensive versus conventional glucose control in critically ill patients." N Engl J Med 360(13): 1283-1297.

Fisk, L. M., A. J. Le Compte, et al. (2012). "STAR development and protocol comparison." IEEE Trans Biomed Eng 59(12): 3357-3364. 
Hann, C. E., J. G. Chase, et al. (2005). "Integral-based parameter identification for long-term dynamic verification of a glucose-insulin system model." Comput Methods Programs Biomed 77(3): 259-270.

Hayashi, N. (2009). "Management of pitfalls for the successful clinical use of hypothermia treatment." J Neurotrauma 26(3): 445-453.

Krinsley, J. S. (2003). "Association between hyperglycemia and increased hospital mortality in a heterogeneous population of critically ill patients." Mayo Clin Proc 78(12): 14711478.

Krinsley, J. S. (2004). "Effect of an intensive glucose management protocol on the mortality of critically ill adult patients." Mayo Clin Proc 79(8): 992-1000.

Le Compte, A. J., D. S. Lee, et al. (2010). "Blood glucose prediction using stochastic modeling in neonatal intensive care." IEEE Trans Biomed Eng 57(3): 509-518.

Lin, J., D. Lee, et al. (2008). "Stochastic modelling of insulin sensitivity and adaptive glycemic control for critical care." Comput Methods Programs Biomed 89(2): 141152.

Lin, J., N. N. Razak, et al. (2011). "A physiological Intensive Control Insulin-NutritionGlucose (ICING) model validated in critically ill patients." Comput Methods Programs Biomed 102(2): 192-205.

McAuley, K. A., J. E. Berkeley, et al. (2011). "The dynamic insulin sensitivity and secretion test--a novel measure of insulin sensitivity." Metabolism 60(12): 1748-1756.

McCowen, K. C., A. Malhotra, et al. (2001). "Stress-induced hyperglycemia." Crit Care Clin 17(1): 107-124.

Mizock, B. A. (2001). "Alterations in fuel metabolism in critical illness: hyperglycaemia." Best Pract Res Clin Endocrinol Metab 15(4): 533-551. 
Neumar, R. W., J. P. Nolan, et al. (2008). "Post-cardiac arrest syndrome: epidemiology, pathophysiology, treatment, and prognostication. A consensus statement from the International Liaison Committee on Resuscitation (American Heart Association, Australian and New Zealand Council on Resuscitation, European Resuscitation Council, Heart and Stroke Foundation of Canada, InterAmerican Heart Foundation, Resuscitation Council of Asia, and the Resuscitation Council of Southern Africa); the American Heart Association Emergency Cardiovascular Care Committee; the Council on Cardiovascular Surgery and Anesthesia; the Council on Cardiopulmonary, Perioperative, and Critical Care; the Council on Clinical Cardiology; and the Stroke Council." Circulation 118(23): 2452-2483.

Penning, S., A. J. Le Compte, et al. (2012). "First pilot trial of the STAR-Liege protocol for tight glycemic control in critically ill patients." Comput Methods Programs Biomed 108(2): 844-859.

Preiser, J. C., P. Devos, et al. (2009). "A prospective randomised multi-centre controlled trial on tight glucose control by intensive insulin therapy in adult intensive care units: the Glucontrol study." Intensive Care Med 35(10): 1738-1748.

Pretty, C., J. G. Chase, et al. (2011). "Impact of glucocorticoids on insulin resistance in the critically ill." Comput Methods Programs Biomed 102(2): 172-180.

Pretty, C. G., A. J. Le Compte, et al. (2012). "Variability of insulin sensitivity during the first 4 days of critical illness: implications for tight glycemic control." Ann Intensive Care 2(1): 17.

Sah Pri, A., J. G. Chase, et al. (2014). "Evolution of insulin sensitivity and its variability in out-of-hospital cardiac arrest (OHCA) patients treated with hypothermia." Crit Care 18(5): 586. 
Taylor, N. A., R. F. Griffiths, et al. (1994). "Epidemiology of hypothermia: fatalities and hospitalisations in New Zealand." Aust N Z J Med 24(6): 705-710.

Thomas, F., C. G. Pretty, et al. (2014). "Reducing the impact of insulin sensitivity variability on glycaemic outcomes using separate stochastic models within the STAR glycaemic protocol." Biomed Eng Online 13: 43.

van den Berghe, G., P. Wouters, et al. (2001). "Intensive insulin therapy in critically ill patients." N Engl J Med 345(19): 1359-1367. 